\title{
What regulates the iron regulator?
}

\author{
Friedrich C. Luft
}

Published online: 24 February 2009

(C) Springer-Verlag 2009

Early in my career, we were pretty ignorant about iron (we did not pump it yet), although we did know a few things. We were aware that the daily intake of iron is (should be) about $10 \mathrm{mg}$ and that $1-2 \mathrm{mg}$ of this iron was absorbed. Iron traveled in the blood bound to a carrier protein called "transferrin" and was stored in various places, such as the reticulo-endothelial system (RES) and bone marrow. As a matter of fact, the court-of-last-appeals for iron deficiency was the staining of bone marrow for iron with "Prussian blue". We were aware that 200 billion erythrocytes were produced daily and that this production would take about $20 \mathrm{mg}$ elemental iron, more than was absorbed. Thus, active recycling had to take place. Total body iron stores were known to be about $5 \mathrm{~g}$ and that half this amount resided in the erythrocytes. We were aware of hereditary hemochromatosis $(\mathrm{HH})$, a favorite examination issue by oral examiners who generally had never ever seen a case either. And we knew about the poor Bantus and their ironcontaining cooking-pots. That was about it, although the iron storage mechanism and the way to measure it, namely ferritin, were introduced about 10 years later. The rest was a mystery, including the other common anemia, termed the "anemia of chronic disease", that not even our smartest professors understood. Fortunately, all that ignorance is behind us, or at any rate, much of it is.

Hepcidin (also known as HFE2) is a hepatic iron regulatory hormone that maintains systemic iron homeostasis. Hepcidin is made by the liver and secreted into the blood stream, where it binds to the iron exporter ferroportin

\section{F. C. Luft $(\square)$}

HELIOS Klinkum-Berlin,

Experimental and Clinical Research Center,

Lindenbergerweg 80 ,

13125 Berlin, Germany

e-mail: luft@charite.de that is located on macrophages, hepatocytes, and intestinal enterocytes. Hepcidin causes ferroportin to be internalized and proteolyzed (chewed up). As a result, hepcidin reduces gastrointestinal iron absorption and macrophage-mediated iron recycling. If hepcidin is so critical to iron-metabolic regulation, then the regulation of hepcidin is critical for our understanding how this very basic portion of human physiology works. In this issue, Casanovas et al. shed light on this question [1]. As is now commonly the case, the understanding of a rare Mendelian disease, $\mathrm{HH}$, led the way. We now know that $\mathrm{HH}$ can be caused by mutations in a membrane protein encoded by a gene that is similar to those coding MHC class I-type proteins. The protein product associates with beta2-microglobulin (beta2M) and is called "HFE" although I am uncertain why. HFE is thought to regulate iron absorption by influencing the interaction of the transferrin receptor with transferrin. The $\mathrm{HH}$ phenotype is also produced by mutations in transferrin receptor 2 (TfR2), hemojuvelin (HJV), or hepcidin itself. Mutations in any of these proteins results in increased duodenal iron absorption, increased macrophage iron release, increased serum iron levels, and liver iron overload.

HJV is a member of the "repulsive guidance molecule" (RGM) family, which also includes the bone-morphogenetic protein (BMP) coreceptors RGMA and DRAGON (RGMB). Babitt et al. [2] reported that HJV is a BMP coreceptor and that HJV mutants associated with the HH phenotype have impaired BMP signaling ability. They showed that BMP upregulates hepatocyte hepcidin expression, a process enhanced by HJV, and that is blunted in hepcidin genedeleted hepatocytes. Their data suggested a mechanism by which hepcidin mutations cause hemochromatosis, namely HJV dysfunction decreases BMP signaling, thereby lowering hepcidin expression. Babbitt et al. [3] next showed that BMP-2 administration increases hepcidin expression and 
decreases serum iron levels in vivo. They also showed that soluble HJV (HJV.Fc) selectively inhibits BMP induction of hepcidin expression in vitro and that administration of HJV.Fc decreases hepcidin expression, increases ferroportin expression, mobilizes splenic iron stores, and increases serum iron levels in vivo. Their data render strong support for a role for modulators of the BMP signaling pathway in treating diseases of iron overload and anemia of chronic disease.

Casanovas et al. [1] show that two highly conserved and sequence-identical BMP-responsive elements located at positions -84/-79 (BMP-RE1) and -2255/-2250 (BMPRE2) of the human hepcidin promoter regulate the basal hepcidin mRNA expression and the hepcidin response to BMP-2 and BMP-6. The authors demonstrate that BMPRE1 and BMP-RE2 show additive effects in responding to HJV-mediated BMP signals. However, only BMP-RE1, which is located in close proximity to a previously identified STAT-binding site, is important for the hepcidin response to IL-6. Their data identifies a connection between the HJV/BMP signaling pathways and hepcidin transcription. Perhaps clinically more interesting is the relationship between inflammation and BMP-dependent hepcidin promoter activation. The added value in this piece of molecular biology is the mechanism how the regulation of hepcidin by HJV comes about. Precise transcriptional control is needed in this pathway. The devil is indeed in the details.

How can we clinicians digest this information? Several scholarly reviews can help [4-8]. Iron-deficiency anemia is the commonest disease worldwide. The anemia of chronic disease is a close second. Pivotal to both is hepcidin. For instance, interleukin-6 and lipopolysaccharide stimulate the hepatic expression of hepcidin, which is an acute phase reactant. Hepcidin inhibits duodenal absorption of iron.
Interferon $\gamma$, lipopolysaccharide, or both can increase the expression of divalent metal transporter 1 (DMT-1) on macrophages and stimulate the uptake of ferrous iron $\left(\mathrm{Fe}^{2+}\right)$. The anti-inflammatory cytokine interleukin-10 upregulates Tfr expression and increases Tfr-mediated uptake of transferrin-bound iron into monocytes. In addition, activated macrophages can then phagocytose and degrade senescent erythrocytes for the recycling of iron, a process that is further induced by tumor necrosis factor (TNF) $\alpha$ through damaging of erythrocyte membranes and stimulation of phagocytosis. Interferon $\gamma$ and lipopolysaccharide down-regulate the expression of the macrophage iron transporter ferroportin 1, thus inhibiting iron export from macrophages, a process that is also affected by hepcidin. At the same time, $\mathrm{TNF} \alpha$, interleukin-1, interleukin-6, and interleukin-10 induce ferritin expression and stimulate the storage and retention of iron within macrophages. These mechanisms lead to a decreased iron concentration in the circulation and thus to a limited availability of iron for erythroid cells. TNF $\alpha$ and interferon $\gamma$ inhibit the production of erythropoietin in the kidney. TNF $\alpha$, interferon $\gamma$, and interleukin-1 directly inhibit the differentiation and proliferation of erythroid progenitor cells. In addition, the limited availability of iron and the decreased biologic activity of erythropoietin lead to inhibition of erythropoiesis and the development of anemia. Thus, the mysterious "anemia of chronic disease" has a molecular basis (Fig. 1).

Missing in this discussion is the "why"? Iron is essential for both host and pathogen, leading to a rational basis for the hypoferremia of infection. However, although downregulation of ferroportin by hepcidin would restrict systemic iron necessary for pathogen growth, cells expressing ferroportin would be particularly vulnerable to infection because of iron retention. This idea has recently been supported by studies of "flatiron" mice, a strain heterozygous for loss-of-

Fig. 1 Hepcidin (by it absence) upregulates iron absorption via enterocytes, controls circulating iron, and influences reticuloendothelial cell macrophages. However, hepcidin (by its presence) downregulates these activities. Hepcidin is upregulated (and therefore downregulates these activites) by ample iron stores or by chronic inflammation. The paradigm encompasses both iron-deficiency anemia and the anemia of chronic inflammatory disease. The figure was adapted from Collins et al. [5]

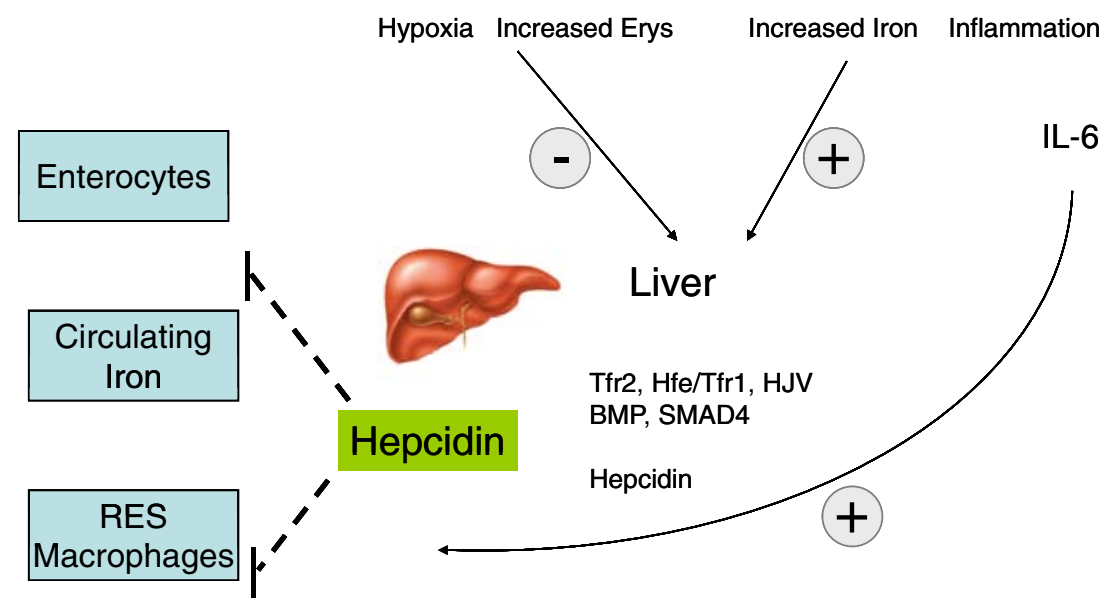


function ferroportin mutations. Compared with control macrophages, flatiron macrophages show increased growth of Chlamydia and Legionella. These data are consistent with observations which demonstrate that ferroportin levels can also influence growth of Salmonella. Hepcidin may even have a direct antimicrobial activity. Thus, the anemia of chronic disease could be a sideshow in a greater picture of innate-immunity protection from infection.

Over 40 years of "clinical experience" (suffering) I can now lay iron-deficiency anemia and the "anemia of chronic disease", not to rest, but to a different pedicle in my clinical experience. Long since dead are the days when a medical house-officer (they do not live in the house any longer anyway) looked at the patients' erythrocytes personally. The new knowledge clarifies the mysteries for us "oldtimers". However, for the "new-comers", who commonly have never seen an erythrocyte (and perhaps with the new hepcidin assay never will), the magnificent increase in knowledge on iron metabolism is an "unearned" privilege.

Respectfully, Friedrich C. Luft

\section{References}

1. Casanovas G, Mleczko-Sanecka K, Altamura S, Hentze MW, Muckenthaler MU (2009) Bone morphogenic protein (BMP)responsive elements located in the proximal and distal hepcidin promoter are critical for its response to $\mathrm{HJV} / \mathrm{BMP} / \mathrm{SMAD}$. J Mol Med. doi:10.1007/s00109-009-0447-2

2. Babitt JL, Huang FW, Wrighting DM, Xia Y, Sidis Y, Samad TA, Campagna JA, Chung RT, Schneyer AL, Woolf CJ, Andrews NC, Lin HY (2006) Bone morphogenetic protein signaling by hemojuvelin regulates hepcidin expression. Nat Genet 38:531-539

3. Babitt JL, Huang FW, Xia Y, Sidis Y, Andrews NC, Lin HY (2007) Modulation of bone morphogenetic protein signaling in vivo regulates systemic iron balance. J Clin Invest 117:19331939

4. Fleming MD (2008) The regulation of hepcidin and its effects on systemic and cellular iron metabolism. Hematology 151158

5. Collins JF, Wessling-Resnick M, Knutons MD (2008) Hepcidin regulation of iron transport. J Nutr 138:2284-2008

6. Weiss G, Goodnough LF (2005) Anemia of chronic disease. N Engl J Med 352:1011-1023

7. Weiss G (2008) Iron metabolism in the anemia of chronic disease. Biochim Biophys Acta

8. Ganz T (2008) Iron homeostatsis: fitting the puzzle pieces together. Cell Metab 7:288-290 